\title{
The Primates 2011 Most-Cited Paper Award is conferred upon Dr. Colin A. Chapman
}

(c) Japan Monkey Centre and Springer 2011

The paper by Dr. Colin A. Chapman (with Dr. Jessica M. Rothman) titled "Within-species differences in primate social structure: evolution of plasticity and phylogenetic constraints" was the most frequently cited of the papers published in Primates between 2008 and 2009. His work thus contributed greatly to enhancing the reputation of our journal. For his achievement, Dr. Chapman will receive gifts from the Japan Monkey Centre, the Primate Society of Japan, and Springer.

17 July 2011

Mitsuo Ichikawa: Executive Director, Japan Monkey Centre

Juichi Yamagiwa: Editor-in-Chief, Primates

Yukio Takahata: President, Primate Society of Japan

Koji Yamashita: Vice President, Springer Japan 